\title{
Quantification of the asymmetric migration of the lipophilic dyes, DiO and DiD, in homotypic co-cultures of chondrosarcoma SW-1353 cells
}

\author{
TOMASZ P. LEHMANN ${ }^{1}$, WOJCIECH JUZWA ${ }^{2}$, KRYSTYNA FILIPIAK $^{3}$, PATRYCJA SUJKA-KORDOWSKA ${ }^{3}$, \\ MACIEJ ZABEL ${ }^{3}$, JAKUB GŁOWACKI ${ }^{4}$, MACIEJ GŁOWACKI ${ }^{5}$ and PAWEŁ P. JAGODZIŃSKI ${ }^{1}$ \\ ${ }^{1}$ Department of Biochemistry and Molecular Biology, Poznan University of Medical Sciences, 60-781 Poznan; \\ ${ }^{2}$ Department of Biotechnology and Food Microbiology, Poznan University of Life Sciences, 60-627 Poznan; \\ ${ }^{3}$ Department of Histology and Embryology, Poznan University of Medical Sciences, 60-781 Poznan; \\ ${ }^{4}$ Medical Center HCP, 61-485 Poznan; ${ }^{5}$ Department of Paediatric Orthopaedics and Traumatology, \\ Poznan University of Medical Sciences, 61-545 Poznan, Poland
}

Received June 3, 2016; Accepted August 15, 2016

DOI: $10.3892 / \mathrm{mmr} .2016 .5793$

\begin{abstract}
DiO and DiD are lipophilic cell labelling dyes used in the staining of cells in vivo and in vitro. The aim of the present study was to quantify the asymmetrical distribution of dyes in co-cultured cells and to measure the intercellular transfer of DiO and DiD. DiO and DiD were applied separately to stain two identical populations of SW-1353 human chondrosarcoma cells that were subsequently co-cultured (homotypic co-culture). The intercellular migration of dyes in the co-cultured cells was measured by flow cytometry and recorded under a fluorescent microscope. DiD and DiO caused no effect on the proliferation of cells, the degradation rate of the two dyes was comparable and crossover effects between dyes were negligible. The results of the present study suggested that asymmetrical intercellular migration of DiD and DiO was responsible for the asymmetrical distribution of these dyes in co-cultured cells. To take advantage of the lipophilic dyes migration in the double-stained co-cultured cells we suggest to apply mixed-dyes controls prior to the flow cytometric analysis. These controls are performed by staining cells with a 1:1 mix of the two dyes and would enable the estimation of the intensity of intercellular contact in co-culture systems. A 1:1 premix of DiO and DiD was applied to estimate cellular effect on intercellular exchange of lipid dyes in co-cultures incubated with cycloheximide and cytochalasin B. The cellular effect contributed $6-7 \%$ of intercellular migration of the lipophilic
\end{abstract}

Correspondence to: Dr Tomasz P. Lehmann, Department of Biochemistry and Molecular Biology, Poznan University of Medical Sciences, ul. Swiecickiego 6, 60-781 Poznan, Poland

E-mail: tlehmann@ump.edu.pl

Key words: carbocyanine lipophilic dyes, chondrosarcoma, co-culture, DiO, DiD, SW-1353 dyes, $\mathrm{DiO}$ and DiD. The majority of the observed intercellular transfer of these dyes was due to non-cellular, passive transfer.

\section{Introduction}

The staining of live cells enables the observation and separation of cells following direct co-culture to analyse any alterations in the phenotypes of co-cultured cells. The application of lipophilic cell labelling dyes from the DiO-family is a powerful tool used for the staining of live cells. These lipophilic fluorescent carbocyanine dyes have been used since the 1980s in vitro and in vivo due to their high quantum efficiency, the simplicity of staining protocols and reduced cytotoxicity compared with hydrophilic dyes $(1,2)$. The excitation and emission spectra of lipophilic fluorescent dyes enables multiple staining (2). Dyes from the DiO-family label the plasma membrane and migrate to cellular organelles as a consequence of membrane turnover (3).

The fluorescence emission spectral overlap between two (or more) fluorochromes may be an issue when performing multicolour experiments. However, modern instruments effectively separate the collected fluorescent signals from various fluorescent dyes by fluorescence overlap compensation (4). Other problems, for example spectral overlap between the emission of one fluorochrome and the excitation of another, are not so readily solved. This overlap is described as the bleed-through effect. To avoid it, care must be taken when selecting fluorochromes and filter sets, or bleed-through must be measured and subtracted from measurements (5). Furthermore, differences in dye stability and/or mobility within the cell or other effects independent of the dye fluorescence emission may influence the results of multicolour experiments. These multicolour experiments followed by flow cytometry may be used to estimate nucleic acid migration between cells. For this, one co-cultured cell population is stained with lipophilic dyes from the DiO family and other cell population is stained with hydrophilic dyes coupled with nucleic acid to monitor their migration (6). Impediments 
resulting from variations in fluorochrome dynamics require consideration when designing multicolour experiments.

$\mathrm{DiO}$ (green) and DiD (red) are used in flow cytometry and confocal microscopy (7-11). It is recommended that various factors be considered when staining with lipophilic dyes, including dye concentration, duration of staining and temperature (12). Our previous study demonstrated the asymmetry of $\mathrm{DiO}$ and DiD distribution in a heterotypic cell co-culture (13). Data concerning the transfer of $\mathrm{DiO}$ or DiD between cells are contradictory; certain authors suggest that lipophilic dyes undergo very low intercellular transfer, whereas others report very high transfer (14-19). As the stable retention of dyes in cells is in question, it is uncertain whether two populations of cells prestained with $\mathrm{DiO}$ and DiD may be separated following co-culture. The size of the co-stained population following co-culture remains to be elucidated.

The aim of the present study was to measure the intercellular migration of dyes in multicolour experiments and quantify their asymmetrical distribution in homotypic co-cultures, following detection by flow cytometry. The optical, chemical and cellular factors involved in the asymmetrical distribution of $\mathrm{DiO}$ and DiD in co-culture experiments were investigated. The results of the present study suggested an application of 1:1 premix of $\mathrm{DiO}$ and DiD to estimate intensity of intercellular contact in co-culture systems.

The data indicating retention of $\mathrm{DiO}$ and $\mathrm{DiD}$ in cultured cells are ambiguous, which precludes the interpretation of results from a number of previous studies (14-19). Due to poor retention and the intercellular migration of lipophilic dyes, separation of cells by cell sorting following co-culture may be hindered. In the present study, two cell lineages were stained separately with $\mathrm{DiO}$ and $\mathrm{DiD}$, before they were mixed and co-cultured in single Petri dishes (direct co-culture system), or in two dishes separated by a $1-\mu \mathrm{m}$ pore membrane (a Transwell indirect co-culture system). By quantifying and comparing the intercellular migration of $\mathrm{DiO}$ and $\mathrm{DiD}$ in the present study, the observed difference in the passive transfer of these two lipophilic dyes demonstrated that the use of these dyes may interfere with cell sorting following co-culture experiments or during dye co-localisation studies.

\section{Materials and methods}

Materials. CHX and CB were purchased from Sigma-Aldrich; Merck Millipore (Darmstadt, Germany). Vybrant ${ }^{\circledR}$ Cell-Labeling solution was obtained from Thermo Fisher Scientific, Inc. (Waltham, MA, USA) and contained the lipophilic dyes, DiO [DiOC18(3); 3,3'-dioctadecyloxacarbocyanine perchlorate] and DiD [DiIC18(5); 1,1'-dioctadecyl-3,3,3', 3'-tetramethylindodicarbocyanine 4-chlorobenzenesulfonate salt], with the following spectral maxima: DiO excitation, $484 \mathrm{~nm} / \mathrm{emission,} 501 \mathrm{~nm}$; and DiD excitation, $644 \mathrm{~nm} / \mathrm{emis}$ sion, $663 \mathrm{~nm}$.

Patients and tissues. Human nucleus pulposus cells (NPCs) and bone marrow mesenchymal stem cells (MSCs) were collected using an anterior approach from four patients undergoing treatment to correct thoracolumbar or lumbar scoliosis during routine preparation of the site for anterior spondylodesis. All patients were recruited into the study consecutively.
The following exclusion criteria were adopted: i) Use of analgesic, antibiotic or steroid medication prior to hospital admission; ii) previous surgery in the spinal area. Patients received in-depth information on the aim of the present study and were assured of anonymity. Informed consent from the legal guardians of each patient was obtained prior to the request to collect NPCs from donors being made. The design of the present study was approved by the Ethics Committee of Poznan University of Medical Sciences (Poznan, Poland; approval number 838/09) and was performed in accordance with universal ethical principles.

The SW-1353 human bone chondrosarcoma cell line was purchased from CLS Cell Lines Service GmbH (Eppelheim, Germany).

Cell culture. For NPCs, the non-degenerate intervertebral disc tissue was dissected primarily from Th12 to L3 to separate the nucleus pulposus from the annulus fibrosus tissue, as described in our previous study (13). Briefly, the nucleus pulposus was enzymatically digested overnight at $37^{\circ} \mathrm{C}$ with $0.02 \%$ collagenase type II (Sigma-Aldrich; Merck Millipore) in serum-free Dulbecco's modified Eagle's medium/Nutrient F-12 Ham (DMEM/F-12; Sigma-Aldrich; Merck Millipore) containing $100 \mathrm{U} / \mathrm{ml}$ penicillin, $100 \mu \mathrm{g} / \mathrm{ml}$ streptomycin and 25 ng/ml amphotericin B (ABAM; Sigma-Aldrich; Merck Millipore). The digested tissue/cell suspension was filtered through sterile nylon fabric to remove remaining tissue debris. The cells were centrifuged at $300 \times \mathrm{g}$ for $5 \mathrm{~min}$ at room temperature, seeded into a tissue culture flask and cultured at $37^{\circ} \mathrm{C}$ in $5 \% \mathrm{CO}_{2} / 95 \%$ air, in $(1: 1 \mathrm{v} / \mathrm{v}) \mathrm{DMEM} / \mathrm{F}-12$ (Sigma-Aldrich; Merck Millipore), supplemented with $10 \%$ foetal bovine serum (Sigma-Aldrich; Merck Millipore), ABAM solution and vitamin C $(5 \mathrm{mg} / \mathrm{ml})$.

For MSCs, the non-degenerative vertebrae were extracted primarily from Th12 to L3. The vertebrae were minced mechanically and enzymatically digested overnight in identical conditions as described for NPCs. The digested tissue/cell suspension was filtered and resuspended in a RosseteSep buffer (Stemcell Technologies, Inc., Vancouver, BC, Canada). Subsequent steps of stem cell enrichment were performed according to the Stem Cells kit procedure (RosetteSep Human Mesenchymal Stem Cell Enrichment Cocktail; Stemcell Technologies, Inc.). Briefly, following overnight digestion, the total lysate was resuspended in the serum-containing medium. Following incubation for $20 \mathrm{~min}$, the cells were placed in Ficoll solution and centrifuged at $300 \times \mathrm{g}$ for $25 \mathrm{~min}$ at room temperature. The intermediate layer of cells was transferred to a cell culture dish and placed in the incubator in medium, as for NPCs except of vitamin C. For the first 4 days of culture the cells were washed daily to enable selection of adhesive cells only. The random subpopulation of MSCs obtained was subcultured in a chondrocyte selective cell culture medium (Human Mesenchymal Stem Cell Functional Identification kit, R\&D Systems, Inc, Minneapolis, MN, USA). The chondrocyte phenotype was confirmed by quantitative polymerase chain reaction, as described in our previous study (13). MSCs destined for co-culture with NPCs were stained with lipophilic dyes and were subsequently cultured in an insert system or in direct co-culture with NPCs. 
SW-1353 cells were seeded onto a tissue culture flask and cultured at $37^{\circ} \mathrm{C}$ in $5 \% \mathrm{CO}_{2} / 95 \%$ air, in (1:1 v/v) DMEM/F-12 supplemented with $10 \%$ foetal bovine serum and ABAM.

Staining cells for flow cytometry and microscopy. For flow cytometry and microscopy, the cells were cultured and stained with DiO or DiD lipophilic dyes. The cells were removed from the culture dish using trypsin (Sigma-Aldrich; Merck Millipore) and were counted. Cells $\left(1 \times 10^{6}\right)$ were incubated for $30 \mathrm{~min}$ at room temperature with $10 \mu 11 \mathrm{mM} \mathrm{DiO}$ or $1 \mathrm{mM}$ DiD dye solutions in $1 \mathrm{ml}$ cell suspension in culture medium [DiO, $10 \mu \mathrm{M}(8.8 \mu \mathrm{g} / \mathrm{ml}) ; \mathrm{DiD}, 10 \mu \mathrm{M}(10.52 \mu \mathrm{g} / \mathrm{ml})]$ and washed twice with phosphate-buffered saline. The cells were subsequently cultured overnight in the culture medium appropriate for the cell type. The following day, the stained cells were subcultured into new culture dishes. Staining of cells directly on culture dishes is recommended by the manufacturer; however, our modified procedure with trypsin detachment was applied to enable mixing of the cells directly following labelling. This was independent of whether trypsin was applied prior to staining or not, and the application of lower concentrations of dyes, fluorescent dyes were observed in the cells rather than in the plasma membranes. A similar protocol was followed for staining of cells by equimolar premixed DiO and DiD; $10 \mu 11 \mathrm{mM} \mathrm{DiO}$ and $10 \mu 11 \mathrm{mM}$ DiD dye solutions were added to $1 \mathrm{ml}$ of cell suspension in culture medium. These cells were cultured and analysed by flow cytometry.

Cells were stained with either $\mathrm{DiD}$ or $\mathrm{DiO}$ and were cultured in the indirect co-culture system using $1 \mu \mathrm{m}$ inserts (translucent PET membrane; BD Biosciences, Franklin Lakes, NJ, USA) and 6-well culture dishes (BD Biosciences). Equal numbers of unstained cells were cultured beneath the inserts. In the direct co-culture system of the double staining experiment, the cells were stained with $\mathrm{DiO}$ or DiD and cultured together in 6-well culture plates at a ratio of 1:1. Following culture, the cells were detached from the plates using trypsin and analysed using a BD FACSAria ${ }^{\mathrm{TM}}$ III (BD Biosciences). The cells designated for observation under a fluorescent microscope $\left(\right.$ EVOS $^{\oplus}$ FL Imaging system; Thermo Fisher Scientific, Inc.) were cultured in 6-well culture plates. Video recordings from this microscope were recorded and are available on request from the corresponding author.

Flow cytometric analysis. The BD FACSAria ${ }^{\mathrm{TM}}$ III flow cytometer is equipped with four lasers $(375,405,488$ and $633 \mathrm{~nm}$ ), 11 fluorescence detectors, and forward scatter (FSC) and side scatter (SSC) detectors. The instrument setup (optical alignment), stability and performance test was performed using the Cytometer Setup and Tracking system (BD Biosciences). FACSFlow solution (BD Biosciences) was used as sheath fluid. The configuration of the flow cytometer was as follows: A $100 \mu \mathrm{m}$ nozzle and $20 \mathrm{psi}(0.138 \mathrm{MPa})$ sheath fluid pressure. The cells were characterized by two non-fluorescent parameters, FSC and SSC, and two fluorescent parameters, green fluorescence from the $\mathrm{DiO}$ reagent was collected using a $530 / 30$ band pass filter (fluorescein isothiocyanate-A detector) and red fluorescence from the DiD reagent was collected using a 660/20 band pass filter (allophycocyanin-A detector). The excitation of the $\mathrm{DiO}$ and $\mathrm{DiD}$ fluorescent reagents was achieved with 488 and $633 \mathrm{~nm}$ lasers, respectively. Flow cytometric analyses were performed using logarithmic gains and specific detector settings. The threshold was set at the FSC signal. The data were acquired in a four-decade logarithmic scale as area signals and analysed with FACSDiva software (version 6.1.3; BD Biosciences). Compensation was performed to ensure that the crossover between the emission spectra of $\mathrm{DiO}$ and DiD caused no effect on the results. Samples for compensation (compensation controls) were cells demonstrating bright emission of individual fluorochromes. The populations were defined by gating in the plots of $\mathrm{DiO}$ vs. $\mathrm{DiD}$ fluorescence signals. Each sample was analysed in triplicate. Cell sorting preceded the doublet discrimination procedure with the use of height vs. width scatter signal measurements in order to discriminate single cells from conglomerates. The cells were sorted into $5 \mathrm{ml}$ cytometric tubes.

The doublet discrimination procedure involves the use of height vs. width scatter signals measurement in order to discriminate single events (single cells) from conglomerates (agglomerated cells). Flow cytometric data are the result of instrument settings prior to, during and following acquisition of the sample. The doublet discrimination procedure uses dot plots for non-fluorescent parameters: Laser-scattered light collected at the front and at a $90^{\circ}$ angle (perpendicular) to the laser beam. Therefore, doublet exclusion may be performed during analysis, even using unstained cells if the condition of the cells is not affected by the staining procedure. Employed prior to sorting, the doublet discrimination procedure provides an element of pure sorting protocol.

Determination of cell viability. SW-1353 cells were stained with $\mathrm{DiO}$ or DiD and cultured for 1,4 or 7 days in 24-well plates $\left(3 \times 10^{4}\right.$ cells/well). Control cells were unstained. Following stimulation, the cells were incubated in serum free DMEM/F-12 containing $0.5 \mathrm{mg} / \mathrm{ml} \mathrm{3-(4,5-dimethylthiazol-2-yl)-2,5-di-}$ phenyltetrazolium bromide (MTT; Sigma-Aldrich; Merck Millipore) for $4 \mathrm{~h}$. The cell culture medium was then removed and a solvent was added ( $0.1 \mathrm{M}$ hydrochloric acid in isopropanol anhydride; Avantor Performance Materials, Gliwice, Poland). The absorbance was read following gentle mixing using the Stat-Fax 2100 microplate reader (Awareness Technology, Inc., Plam City, FL, USA) at a wavelength of $492 \mathrm{~nm}$ (background absorbance was measured at a wavelength of $630 \mathrm{~nm}$ ).

Statistical analysis. The data are expressed as the mean \pm standard deviation of at least three biological repeats (staining and co-culture). The groups were compared using the Student's t-test and the STATISTICA data analysis software program (version 12.0; StatSoft, Inc., Tulsa, OK, USA). P $<0.05$ was considered to indicate a statistically significant difference.

\section{Results}

Asymmetric distribution of dyes following direct co-culture. Lassailly et al (15) observed an extensive transfer of DiO-type lipophilic dyes from leukemic cells to neighbouring cells in vitro and in vivo, and called this phenomenon 'microenvironmental contamination'. Strassburg et al (16), in their study of co-cultured human MSCs and NPCs, observed that up to $87 \%$ of unlabelled cells demonstrated DiL 

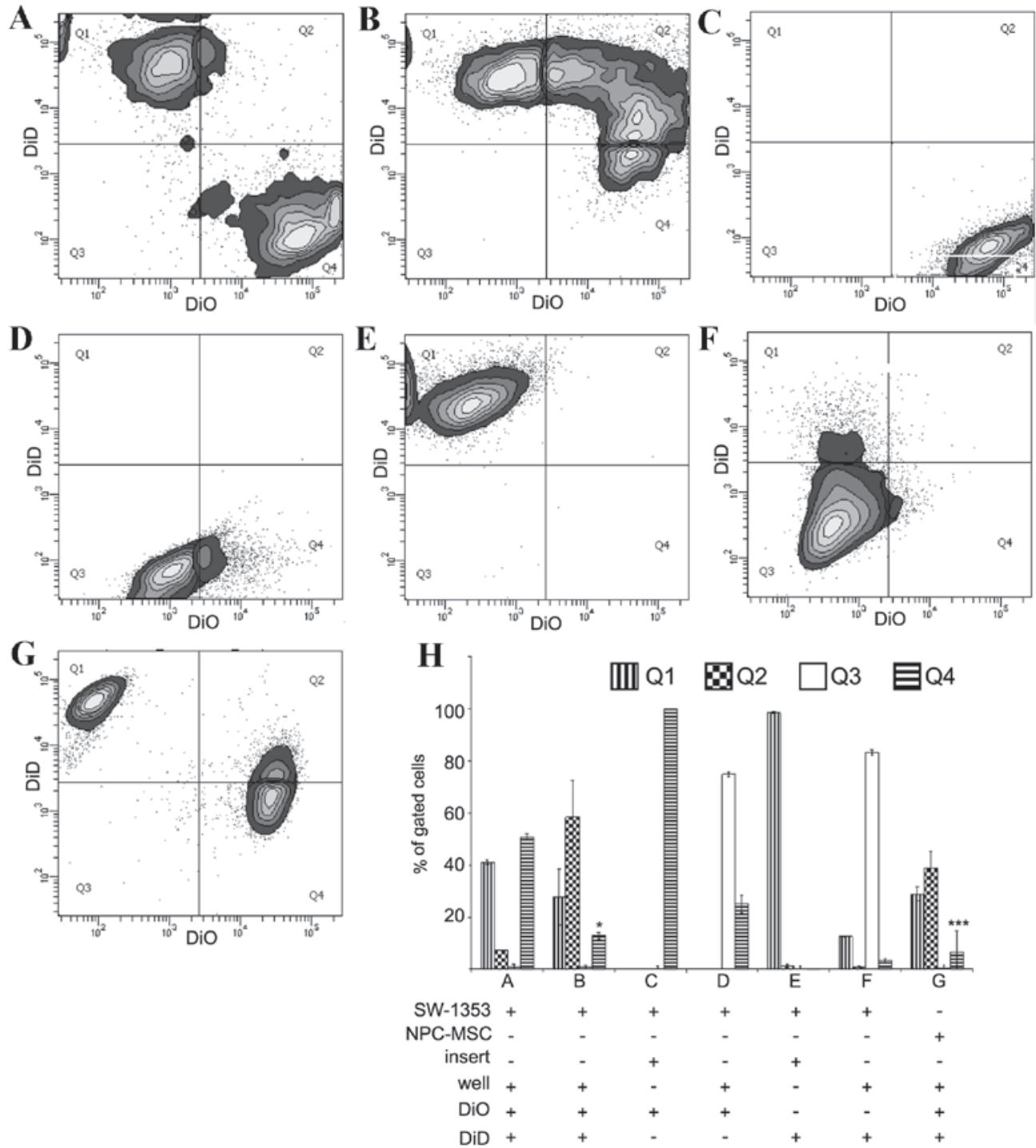

Figure 1. Transfer of DiO and DiD in co-culture systems of SW-1353 cells, NPCs and MSCs. The representative contour plots were obtained by FACS analysis of co-cultured cells. Harvested cells were excited by blue $(488 \mathrm{~nm})$ and red $(633 \mathrm{~nm})$ lasers corresponding to DiO and DiD, respectively. The emission signals were detected by $530 / 30$ and 660/20 nm detectors. In the direct co-culture system 200,000 DiO-labelled SW-1353 cells were mixed with 200,000 DiD-labelled SW-1353 cells. The cells were measured by FACS (A) immediately following mixing or (B) following four days of co-culture. In addition, FACS was performed following 4 days of indirect co-culture of (C) 200,000 DiO-labelled cells in a well insert and (D) 200,000 unstained cells in the bottom well, and (E) 200,000 DiD-labelled cells in a well insert and (F) 200,000 unstained cells in the bottom well. (G) For comparison, 200,000 DiO-labelled NPCs were mixed with 200,000 DiD-labelled MSCs and co-cultured in the direct co-culture system for 4 days. (H) Quantification of three repeats of FACS analyses expressed as the percentage of gated cells. The gating of the flow cytometry system was set to cover the mean distribution of DiD-stained cells (Q1), double-stained cells (Q2), unstained controls $(\mathrm{Q} 3)$ and DiO-stained cells $(\mathrm{Q} 4)$. The data are expressed as the mean \pm standard deviation $\left({ }^{*} \mathrm{P}<0.05\right.$ in $\mathrm{B}$ showing $\mathrm{Q} 1$ vs. Q4; ${ }^{* * * *} \mathrm{P}<0.001$ in G showing Q1 vs. Q4). NPCs, nucleus pulposus cells; MSCs, mesenchymal stem cells; FACS, fluorescent-activated cell sorting.

fluorescence following 7 days. Therefore, DiO-type lipophilic dyes may be transferred and incorporated by unlabelled cells. Our previous studies revealed a similar phenomenon of $\mathrm{DiO}$ and DiD transfer between MSCs and NPCs in heterotypic co-culture (13). Using a fluorescent microscope, rapid movements of $\mathrm{DiO}$ and $\mathrm{DiD}$ particles in SW-1353 cells were detected (data not shown; video recording available on request from the corresponding author).

In our previous study, a predominance of DiD-stained cells over DiO-stained cells was observed by flow cytometry, which we termed 'Q1-Q4 asymmetry' (13). In the present study, homotypic co-cultures of SW-1353 cells were performed to precisely quantify the Q1-Q4 asymmetry. Unstained SW-1353 cells were subcultured and digested using trypsin. This was followed by separate staining with $\mathrm{DiD}$ and $\mathrm{DiO}$. The DiDand DiO-stained cells were subsequently mixed 1:1 with a combination of 200,000 cells from each coloured population for direct co-culture in one well of a 6-well culture dish for 4 days. The DiD- or DiO stained cells were also placed unmixed in the upper dish $(200,000$ stained cells) of the Transwell insert system for 4 days. There were 200,000 unstained cells in the lower dish of the Transwell system. The cells were subsequently analysed by flow cytometry, with the gating of the system set to cover the mean distribution of DiD-stained cells $(\mathrm{Q} 1)$, double-stained cells $(\mathrm{Q} 2)$, unstained controls $(\mathrm{Q} 3)$ and DiO-stained cells (Q4). The properties of the dyes' interaction with the cells induced a wide dispersion of events and certain events exceeded the boundaries established for gating. 
A<smiles>CCN1C(=CC=Cc2oc3ccccc3[n+]2CC)Oc2ccccc21</smiles>

B

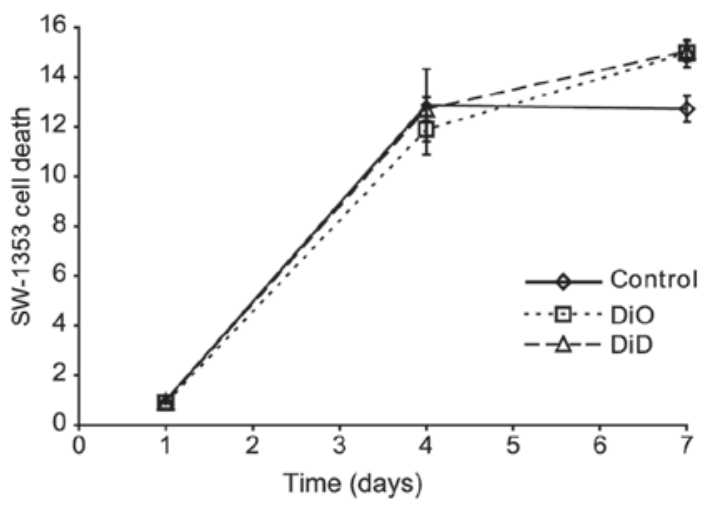<smiles>CCN1C(=CC=CC2=[N+](CC)c3ccccc3C2(C)C)C(C)(C)c2ccccc21</smiles>

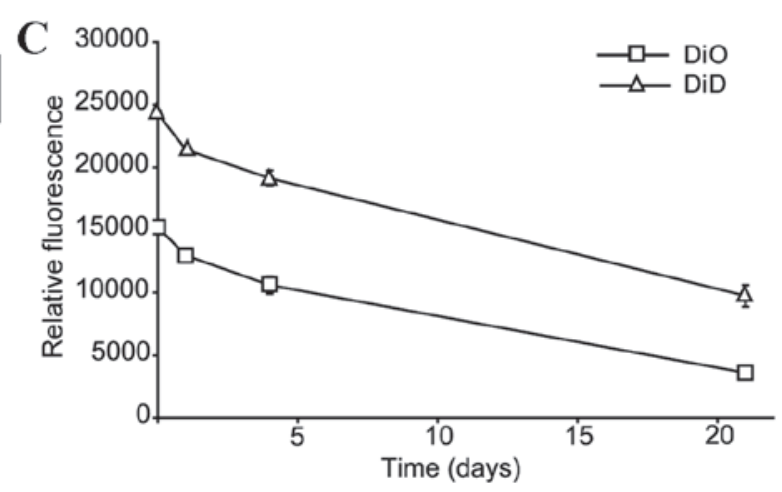

Figure 2. Effect of DiO and DiD on SW-1353 cells. (A) Chemical structures of DiO and DiD. (B) The viability of SW-1353 cells stained with DiO and DiD was determined. SW-1353 cells were stained with DiO (dotted line) or DiD (dashed line) and cultured for 1, 4 or 7 days in $24-$ well plates. Control cells (solid line) were unstained. Following incubation, a 3-(4,5-dimethylthiazol-2-yl)-2,5-diphenyltetrazolium bromide assay was performed. DiO and DiD labelling caused no affect on cell viability. The data are expressed as the mean \pm standard deviation. (C) The kinetics of DiO and DiD fluorescence in SW-1353 cells was determined. SW-1353 cells $(200,000)$ were stained with DiO or DiD, mixed and co-cultured in the direct system for 1 , 4 or 21 days. Fluorescence in quadrants Q1 (DiD) and Q4 (DiO) was measured by FACS and expressed as the mean \pm standard deviation. Although DiD had a greater fluorescence intensity throughout, the dyes degraded at similar rates.

Asymmetry of the distribution of events was observed in the flow cytometric cytograms (Fig. 1A and B). There was double the percentage of events (ratio 2:1) corresponding to DiD-stained cells in the Q1 quartile compared with DiO-stained in the Q4 quartile following 4 days of direct co-culture of SW-1353. FACS analysis of SW-1353 cells co-cultured in the Transwell system revealed that without direct contact of cells the transfer of $\mathrm{DiO}$ and DiD was $<50 \%$ of the transfer in the direct co-culture (Fig. 1C and F).

Direct co-culture of MSCs stained with DiD and NPCs stained with DiO resulted in Q1-Q4 asymmetry, with the ratio of Q1:Q4 being 4:1 (Fig. 1G). Quantification of the percentages of cells in each quadrant from each experiment is presented in Fig. 1H. The Q1-Q4 asymmetry may be produced by various factors, including an impact of the dyes on cell viability, a rate of dye degradation, a crossover of emission/excitation spectra, the intercellular mobility of dyes and chemical structure. The chemical structures of DiD and DiO are presented in Fig. 2A. The following experiments were performed to address these issues.

Differences in cell viability are not responsible for asymmetry. SW-1353 cells were stained with DiO or DiD and cultured (25,000 cells/well) for 1,4 or 7 days in 24 -well plates. The cells were subsequently analysed using an MTT assay. No differences in viability were observed between cells stained with DiO and DiD (Fig. 2B).
Degradation rates of $\mathrm{DiO}$ and DiD are not responsible for asymmetry. To estimate the relative difference in the degradation rate of $\mathrm{DiO}$ and $\mathrm{DiD}, \mathrm{SW}-1353$ cells were stained separately with the lipophilic dyes (Fig. 2C). The stained cells were mixed at a ratio of $1: 1$ and cultured for 1,4 or 21 days. The mean fluorescence of the cells was measured by flow cytometry. Although $\mathrm{DiO}$ florescence intensity was lower compared with DiD fluorescence from the beginning of the experiment, the fluorescence decreased at a similar rate (Fig. 2C). Therefore, the degradation rates of the fluorescence were similar. Following 4 days of culture a negligible $3.4 \%$ faster decrease of fluorescence intensity was measured for $\mathrm{DiO}$ compared with for $\mathrm{DiD}(\mathrm{P}=0.0216)$. Therefore, the Q1-Q4 asymmetry is not explained by the reduced degradation of DiD.

Crossover of emission-excitation spectra is not responsible for asymmetry. Compensation using single stained cells was applied to eliminate any emission-excitation crossover that may have been responsible for the Q1-Q4 asymmetry in the DiD- and DiO-stained cells. Additionally the SW-1353 cells were analysed using independent excitation with $488 \mathrm{~nm}$ or $630 \mathrm{~nm}$ lasers and routine application of lasers excitation (Table I). In this experiment, an unexpectedly high emission of light in the 660/20 detector was observed. SW-1353 cells stained with DiD and excited by 488 and $630 \mathrm{~nm}$ lasers exhibited quadrupled emission in comparison with the single 
Table I. Mean fluorescence intensities.

\begin{tabular}{|c|c|c|c|c|c|c|}
\hline \multirow[b]{2}{*}{ Experiment } & \multicolumn{2}{|c|}{ Dye } & \multicolumn{2}{|c|}{ Excitation } & \multicolumn{2}{|c|}{ Emission (RFU) } \\
\hline & $\mathrm{DiO}$ & DiD & $488 \mathrm{~nm}$ & $633 \mathrm{~nm}$ & $530 / 30$ & $660 / 20$ \\
\hline 1 & + & - & + & - & 10,760 & 0 \\
\hline 2 & + & - & - & + & 0 & 152 \\
\hline 3 & - & + & + & - & 116 & 0 \\
\hline 4 & - & + & - & + & 0 & 26,611 \\
\hline 5 & + & - & + & + & 10,245 & 164 \\
\hline 6 & - & + & + & + & 0 & 108,692 \\
\hline 7 & + & + & + & - & 3,337 & 0 \\
\hline 8 & + & + & - & + & 0 & 65,204 \\
\hline 9 & + & + & + & + & 11,364 & 94,900 \\
\hline
\end{tabular}

All results were obtained by fluorescent activated cell sorting analysis (1) of SW-1353 cells stained with $\mathrm{DiO}$ and/or $\mathrm{DiD}$. DiO and $\mathrm{DiD}$ stained cell populations were separately excited by blue $(488 \mathrm{~nm})$ or red $(633 \mathrm{~nm})$ lasers and with the two lasers. The emission signals were detected by $530 / 30$ and $660 / 20 \mathrm{~nm}$ detectors corresponding to $\mathrm{DiO}$ and $\mathrm{DiD}$ respectively. +, dye/laser was applied in the experiment; -, dye/laser was excluded in the experiment; RFU, relative fluorescent units.

excitation by the $488 \mathrm{~nm}$ laser. However, this optical effect, which greatly enhanced the emission of DiD, caused no affect on the counting of events and, therefore, did not explain Q1-Q4 asymmetry.

Greater mobility of DiD may be responsible for Q1-Q4 asymmetry. The above experiments eliminated the most predictable reasons for Q1-Q4 asymmetry, with the exception of the unequal transfer rates of $\mathrm{DiO}$ and $\mathrm{DiD}$. Following 4 days of co-culture, two-thirds of the SW-1353 cells, which had formerly been stained with $\mathrm{DiO}$ or $\mathrm{DiD}$ and mixed at a ratio of 1:1, were double-stained (Q2). This occurred due to the intercellular transfer of dyes, rather than their degradation, which would be observed as an increase of events in Q3. Therefore, Q1-Q4 asymmetry may be caused by the greater mobility of DiD compared with DiO. This unequal rate of transfer greatly complicates the interpretation of results from experiments involving double-staining in homotypic and heterotypic co-cultures. This transfer of dyes maybe cell-dependent or independent.

To determine whether the diffusion of lipophilic dyes is a specific and cell-dependent phenomenon, a protein synthesis blocker, $\mathrm{CHX}$, and actin filaments polymerisation inhibitor, CB, were added to the cell culture (Fig. 3). SW-1353 cells stained separately with $\mathrm{DiO}$ and DiD were mixed (200,000 cells from each population) and co-cultured directly in the absence (control) or presence of CHX $(10 \mu \mathrm{g} / \mathrm{ml})$ or CB $(350 \mathrm{nM})$. In addition, SW-1353 cells were stained with an equimolar mix of $\mathrm{DiO}$ and $\mathrm{DiD}$, and were cultured without $\mathrm{CHX}$ or $\mathrm{CB}$. Following 4 days, the cells were analysed by flow cytometry. The controls revealed a similar staining pattern as shown in Fig. 1 (Fig. 3A). The 'equimolar population' served to outline a subpopulation of Q2 labelled PQ4 (Fig. 3B). This narrow subpopulation displayed a linear distribution of events with a range of two orders of magnitude of fluorescence intensity. Taking PQ4 subpopulation as a reference, the present study observed that in cultures treated with $\mathrm{CHX}$ and $\mathrm{CB}$ PQ4 subpopulations were $\sim 1 / 3$ smaller compared with the control (control 19.7, CHX 12.8 and CB 14.0\%, respectively; Fig. 3A, $\mathrm{C}$ and D). Differences in the number of events in control Q2, CHX Q2 and CB Q2 were not significant. The use of cells stained with a balanced ratio of dyes may be the additional control in flow cytometry for multicolour experiments that aim to measure dynamics in intercellular contact.

\section{Discussion}

Various successful co-culture methodologies to investigate heterotypic cell-cell interactions in mixed co-cultures are noted in previous studies (20). Certain of these methodologies employed lipophilic dyes for tracking co-cultured cells (2). These dyes present numerous advantages; however, they have various properties that limit their use in the observation and separation of co-cultured cells. One of the limiting factors is intercellular migration of lipophilic dyes (15). In our previous study, it was observed that this migration was asymmetrical in the co-culture of NPCs and MSCs stained by DiO and DiD (13). However, this previous study did not demonstrate quantitative differences in the intercellular transfer of the two lipophilic dyes, $\mathrm{DiO}$ and $\mathrm{DiD}$, in homotypic cell co-culture systems. These differences may be measured by flow cytometry and are indistinguishable for fluorescent microscopy. In the present study, using flow cytometry, differences in the migration of $\mathrm{DiO}$ and $\mathrm{DiD}$ were measured. The difference was expressed as a number of events in gates Q1 and Q4, corresponding to DiD and DiO-stained cells and termed 'Q1-Q4 asymmetry'. SW-1353 human chondrosarcoma cells were stained with $\mathrm{DiO}$ and $\mathrm{DiD}$, and were subsequently co-cultured directly and indirectly. SW-1353 cells were selected for the purposes of the present study, despite their limited similarity to human articular chondrocytes (21). Compared with primary cells, SW-1353 cells exhibited increased genetic and phenotypic homogeneity, which is required for quantification during flow cytometry analyses. Comparable asymmetry has been 

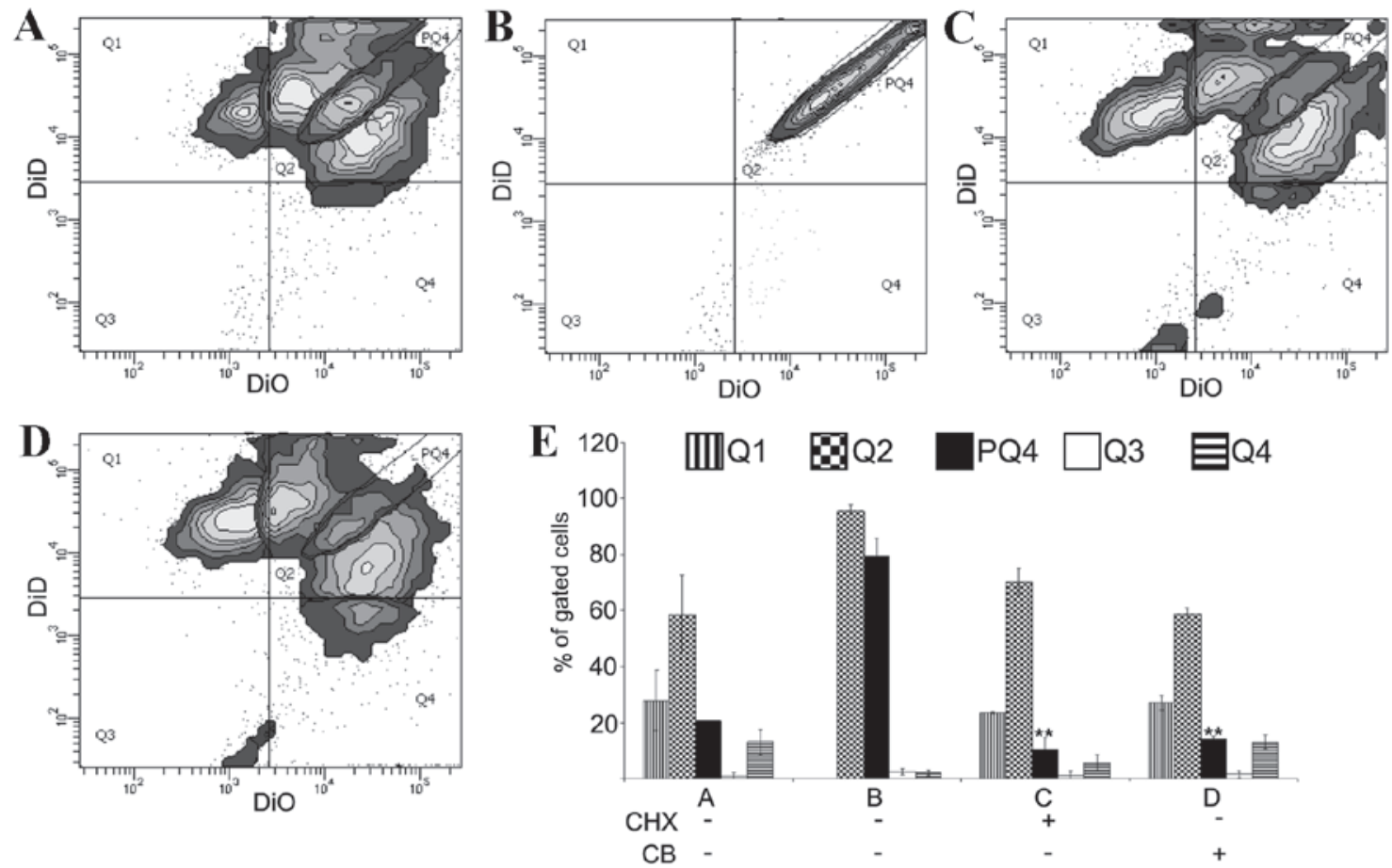

Figure 3. Intercellular migration of DiO and DiD in SW-1353 cells. The representative contour plots were obtained by FACS analysis of co-cultured SW-1353 cells. They were excited by blue $(488 \mathrm{~nm})$ and red $(633 \mathrm{~nm})$ lasers corresponding to $\mathrm{DiO}$ and $\mathrm{DiD}$, respectively. The emission signals were detected by $530 / 30$ and 660/20 nm detectors. (A) In the direct co-culture system, 200,000 DiO-labelled SW-1353 cells were mixed with 200,000 DiD-labelled cells and were measured by FACS following 4 days of co-culture. (B) Control cells were stained by equimolar premixed $\mathrm{DiO}$ and DiD. The cells were treated with (C) $10 \mu \mathrm{g} / \mathrm{ml}$ cycloheximide or (D) $350 \mathrm{nM}$ cytochalasin B. (E) Quantification of FACS analyses were expressed as percentage of gated cells. Q1, Q2, Q3 and Q4 represent quadrants in cytograms. PQ4 represents subpopulation of Q2 population subgated upon analysis of cells stained by premixed DiO and DiD. The data are expressed as the mean \pm standard deviation ( $(" \mathrm{P}<0.05$ in $\mathrm{PQ} 4 \mathrm{~A}$ vs. PQ4 C, and PQ4 A vs. PQ4 D). CHX, cycloheximide; CB, cytochalasin B.

observed in NP-MSC co-culture, therefore asymmetry may be considered a cell-independent process. However, this requires further investigation in other cell types.

Using the direct co-culture system of SW-1353 cells stained separately by $\mathrm{DiO}$ and $\mathrm{DiD}$, mixed at a ratio of 1:1, a $2: 1$ ratio of DiD-stained cells to DiO-stained cells was observed following 4 days of co-culture. These results indicated that this asymmetry was caused by the imbalance in intercellular migration of $\mathrm{DiO}$ and $\mathrm{DiD}$.

The present study supported earlier data demonstrating the intercellular transfer of the $\mathrm{DiO}$ family of dyes mediated by cell-cell contact and diffusible particles $(15,16)$. Investigation of DiI transport from labelled to unlabelled cells following 7 days of co-culture revealed that $80 \%$ of the unlabelled cells became stained (16).

The observed Q1-Q4 asymmetry is unlikely to be caused by an effect on cell proliferation, as $\mathrm{DiD}$ and $\mathrm{DiO}$ labelling did not affect proliferation. The degradation rate of the two dyes was comparable and crossover effects were negligible. Through the elimination of these possible explanations, the results of the present study indicated that the Q1-Q4 asymmetry was caused by varying diffusion rates of $\mathrm{DiD}$ and $\mathrm{DiO}$. These dyes have different chemical structures, in addition DiD solution contains 4-chlorobenzenesulfonate and ethanol. DiO solution contains dimethylformamide. It has been identified that in DiD-perchlorate (DiD without 4-chlorobenzenesulfonate) the diffusion properties are comparable to those of DiI; however, DiD-perchlorate diffusion was not evaluated in association with $\mathrm{DiO}(22)$.
The Q1-Q4 asymmetry hindered confirmation of whether the cell-dependent or -independent process induced microenvironmental contamination. The gating of the PQ4 sub-population obtained by analysis of the double-stained SW-1353 cells by 1:1 DiO/DiD premix enabled this confirmation. By incubating SW-1353 with CHX and CB, it was revealed that dye transfer is primarily a cell-independent process.

This double-prestained population is important as a control; otherwise the Q1-Q4 asymmetry impedes precise quantification of the intercellular transfer of dyes used in dual-staining. A strict control must be applied prior to each experiment involving double staining of cells. The control staining must be performed by adding the mixed dyes to each cell line.

The applicability of DiO and DiD in the separation of co-cultured cells by flow cytometry requires additional investigation. Although DiO and DiD are referred to as "lipophilic dyes' intense internalization and poor persistence in the cell membrane was observed. However, these lipophilic dyes may be a useful tool in quantification of intercellular interactions. Lipophilic molecules, including $\mathrm{DiO}$ and $\mathrm{DiD}$, characterised by subtle differences in biocompatibility, may be a model of the intercellular traffic of similar endogenous molecules produced by two cell populations.

In conclusion, the present study quantified the asymmetry of DiO and DiD distribution in SW-1353 homotypic co-cultures, and determined that this asymmetry is cell-independent, and is not explained by cell proliferation, dye degradation or crossover. The limitation of the present study is the undetermined time for the complete double staining of the 
whole population of co-cultured cells by $\mathrm{DiO}$ and $\mathrm{DiD}$. The uninvestigated role of 4-chlorobenzenesulfonate may be one of the factors causing the different rate of release of $\mathrm{DiO}$ and DiD from the cells. Therefore, it will be useful to apply dyes with the same additives and analyse migration. Future studies are required to confirm the hypothesis that the speed of $\mathrm{DiO}$ and DiD migration is different, by measuring the movement of dye particles visible under microscope. This approach of using software to analyse video recordings may confirm the differences in the speed of DiO and DiD transport. Finally, the type of cellular activity responsible for active dye transfer requires clarification.

\section{Acknowledgements}

The present study was supported solely by the Polish National Science Centre (grant no. NN403600538). The authors would like to thank Ms. Beata Raczak and Ms. Bogumila Ratajczak (Department of Biochemistry and Molecular Biology, Poznan University of Medical Sciences, Poznan, Poland) for their indispensable assistance during the preparation of this paper.

\section{References}

1. Honig MG and Hume RI: Fluorescent carbocyanine dyes allow living neurons of identified origin to be studied in long-term cultures. J Cell Biol 103: 171-187, 1986.

2. Progatzky F, Dallman MJ and Lo Celso C: From seeing to believing: Labelling strategies for in vivo cell-tracking experiments. Interface Focus 3: 20130001, 2013.

3. Sezgin E, Chwastek G, Aydogan G, Levental I, Simons K and Schwille P: Photoconversion of bodipy-labeled lipid analogues. Chembiochem 14: 695-698, 2013.

4. Shapiro HM: Practical Flow Cytometry 736, 2003.

5. Rietdorf J and Stelzer EHK: Special optical elements. In: Handbook of Biological Confocal Microscopy. New York, Springer Verlag, 2006

6. Thayanithy V, Dickson EL, Steer C, Subramanian S and Lou E: Tumor-stromal cross talk: Direct cell-to-cell transfer of oncogenic microRNAs via tunneling nanotubes. Transl Res 164: 359-365, 2014.

7. Fritzsch B, Muirhead KA, Feng F, Gray BD and Ohlsson-Wilhelm BM: Diffusion and imaging properties of three new lipophilic tracers, NeuroVue Maroon, NeuroVue Red and NeuroVue Green and their use for double and triple labeling of neuronal profile. Brain Res Bull 66: 249-258, 2005.
8. Huerta L, López-Balderas N, Larralde C and Lamoyi E: Discriminating in vitro cell fusion from cell aggregation by flow cytometry combined with fluorescence resonance energy transfer. J Virol Methods 138: 17-23, 2006.

9. Lo Celso C, Fleming HE, Wu JW, Zhao CX, Miake-Lye S, Fujisaki J,CôtéD, Rowe DW,Lin CP and Scadden DT: Live-animal tracking of individual haematopoietic stem/progenitor cells in their niche. Nature 457: 92-96, 2009.

10. Maklad A and Fritzsch B: The developmental segregation of posterior crista and saccular vestibular fibers in mice: A carbocyanine tracer study using confocal microscopy. Brain Res Dev Brain Res 135: 1-17, 2002.

11. Plotnikov EY, Khryapenkova TG, Galkina SI, Sukhikh GT and Zorov DB: Cytoplasm and organelle transfer between mesenchymal multipotent stromal cells and renal tubular cells in co-culture. Exp Cell Res 316: 2447-2455, 2010.

12. Jensen EC: Use of fluorescent probes: Their effect on cell biology and limitations. Anat Rec (Hoboken) 295: 2031-2036, 2012

13. Lehmann TP, Filipiak K, Juzwa W, Sujka-Kordowska P, Jagodziński PP, Zabel M, Głowacki J, Misterska E, Walczak M and Głowacki M: Co-culture of human nucleus pulposus cells with multipotent mesenchymal stromal cells from human bone marrow reveals formation of tunnelling nanotubes. Mol Med Rep 9: 574-582, 2014.

14. Burguera EF, Bitar M and Bruinink A: Novel in vitro co-culture methodology to investigate heterotypic cell-cell interactions. Eur Cell Mater 19: 166-179, 2010.

15. Lassailly F, Griessinger E and Bonnet D: 'Microenvironmental contaminations' induced by fluorescent lipophilic dyes used for noninvasive in vitro and in vivo cell tracking. Blood 115: 5347-5354, 2010

16. Strassburg S, Hodson NW, Hill PI, Richardson SM and Hoyland JA: Bi-directional exchange of membrane components occurs during co-culture of mesenchymal stem cells and nucleus pulposus cells. PLoS One 7: e33739, 2012.

17. Yumoto K, Berry JE, Taichman RS and Shiozawa Y: A novel method for monitoring tumor proliferation in vivo using fluorescent dye DiD. Cytometry A 85: 548-555, 2014.

18. Cirulis JT, Strasser BC, Scott JA and Ross GM: Optimization of staining conditions for microalgae with three lipophilic dyes to reduce precipitation and fluorescence variability. Cytometry A 81: 618-626, 2012.

19. Fonseca PC, Nihei OK, Savino W, Spray DC and Alves LA: Flow cytometry analysis of gap junction-mediated cell-cell communication: Advantages and pitfalls. Cytometry A 69: 487-493, 2006.

20. Goers L, Freemont P and Polizzi KM: Co-culture systems and technologies: Taking synthetic biology to the next level. J R Soc Interface 11: pii: 20140065, 2014.

21. Gebauer M, Saas J, Sohler F, Haag J, Söder S, Pieper M, Bartnik E, Beninga J, Zimmer R and Aigner T: Comparison of the chondrosarcoma cell line SW1353 with primary human adult articular chondrocytes with regard to their gene expression profile and reactivity to IL-1beta. Osteoarthritis Cartilage 8: 697-708, 2005.

22. Agmon A, Yang LT, Jones EG and O'Dowd DK: Topological precision in the thalamic projection to neonatal mouse barrel cortex. J Neurosci 15: 549-561, 1995. 